\title{
Graphs and Degree Equitability
}

\author{
Ahmad N. Al-Kenani ${ }^{1}$, Nandappa D. Soner ${ }^{2}$, Anwar Alwardi ${ }^{2}$ \\ ${ }^{1}$ Department of Mathematics, King Abdulaziz University, Jeddah, Saudi Arabia \\ ${ }^{2}$ Department of Studies in Mathematics, University of Mysore, Mysore, India \\ Email: aalkenani10@hotmail.com, soner@maths.uni-mysore.ac.in, a_wardi@hotmail.com
}

Received March 5, 2013; revised April 5, 2013; accepted April 14, 2013

Copyright (C) 2013 Ahmad N. Al-kenani et al. This is an open access article distributed under the Creative Commons Attribution License, which permits unrestricted use, distribution, and reproduction in any medium, provided the original work is properly cited.

\begin{abstract}
Let $G=(V, E)$ be a graph. If $\varphi$ is a function from the vertex set $V(G)$ to the set of positive integers. Then two vertices $u, v \in V(G)$ are $\varphi$-equitable if $|\varphi(u)-\varphi(v)| \leq 1$. By the degree, equitable adjacency between vertices can be redefine almost all of the variants of the graphs. In this paper we study the degree equitability of the graph by defining equitable connectivity, equitable regularity, equitable connected graph and equitable complete graph. Some new families of graphs and some interesting results are obtained.
\end{abstract}

Keywords: Equitable Domination Number; Equitable Path; Equitable Walk; Equitable Connected Graph; Equitable Regular Graph; Equitable Complement Graph; Equitable Cut Vertex; Equitable Line Graph

\section{Introduction}

All the graphs considered here are finite and undirected with no loops and multiple edges. As usual $p=|V|$ and $q=|E|$ denote the number of vertices and edges of a graph $G$, respectively. In general, we use $\langle X\rangle$ to denote the subgraph induced by the set of vertices $X$ and $N(v)$ and $N[v]$ denote the open and closed neighbourhoods of a vertex $v$, respectively. The degree of the vertex $v$ in $G$ is denoted by $\operatorname{deg}_{G}(v)$ or $\operatorname{deg}(v)$. For graph theoretic terminology, we refer to Harary [1]. The degree equitable domination has been studied in [2]. A subset $D$ of $V(G)$ is called an equitable dominating set of a graph $G$ if for every $v \in V-D$, there exists a vertex $u \in D$ such that $u v \in E(G)$ and

$|\operatorname{deg}(u)-\operatorname{deg}(v)| \leq 1$. The minimum cardinality of such a dominating set is denoted by $\gamma_{e}$ and is called the equitable domination number of $G$. The set $D$ is minimal if for any vertex $u \in D, D-\{u\}$ is not an equitable dominating set of $G$. The equitable neighbourhood of $u$ denoted by $N_{e}(u)$ is defined as

$$
\begin{aligned}
& N_{e}(u)=\{v \in V|v \in N(u),| \operatorname{deg}(u)-\operatorname{deg}(v) \mid \leq 1\} \text { and } \\
& u \in I_{e} \Leftrightarrow N_{e}(u)=\phi . \text { The cardinality of } N_{e}(u) \text { is de- } \\
& \text { noted by } \operatorname{deg}_{e}(u) \text { and it is called equitable degree of } \\
& \text { the vertex } u \text { in } G \text {. The maximum and minimum equi- } \\
& \text { table degree of } G \text { are denoted respectively by } \Delta_{e}(G) \\
& \text { and } \delta_{e}(G) \text {. That is } \Delta_{e}(G)=\max _{u \in V(G)}\left|N_{e}(u)\right|,
\end{aligned}
$$

$\delta_{e}(G)=\min _{u \in V(G)}\left|N_{e}(u)\right|$. An edge $e=u v$ is called an equitable edge if $|\operatorname{deg}(u)-\operatorname{deg}(v)| \leq 1$. A subset $S$ of $V$ is called an equitable independent set, if $S$ contains no vertices $u, v$ such that $v \in N_{e}(u)$. If a vertex

$u \in V$ satisfies $|\operatorname{deg}(u)-\operatorname{deg}(v)| \geq 2$ for all $v \in N(u)$, then $u$ is in the equitable dominating set. Such vertices are called equitable isolated vertices.

Let $G$ be a simple graph with $n$ vertices $v_{1}, v_{2}, \cdots, v_{n}$. Then its adjacency matrix $A=\left[a_{i j}\right]$ is a $n \times n$ matrix whose entries $a_{i j}$ are given by

$$
a_{i j}= \begin{cases}1, & \text { if } v_{i} \text { and } v_{j} \text { are adjacent; } \\ 0, & \text { otherwise. }\end{cases}
$$

In the same way, the degree equitable adjacency matrix denoted by $A_{e}=\left[b_{i j}\right]$ is a $n \times n$ matrix whose entries $b_{i j}$ are given by

$$
b_{i j}= \begin{cases}1, & \text { if } v_{i} \text { and } v_{j} \text { are equitable adjacent } ; \\ 0, & \text { otherwise. }\end{cases}
$$

where The equitable adjacency between any two vertices $u, v$ in $V$ is defined as follows: the vertex $v$ is equitable adjacent to $u$ if and only if $u$ is adjacent to $v$ and also $\left|\operatorname{deg}_{e}(v)-\operatorname{deg}_{e}(u)\right| \leq 1$.

Degree equitable adjacency has interesting applications in the context of social networks. In a network, 
nodes with nearly equal capacity may interact with each other in a better way. In society, persons with nearly equal status, tend to be friendly. In industry, employees with nearly equal powers form associations and move closely. Equitability among citizens in terms of wealth, health, status, etc is the goal of a democratic nation. These ideas motivated us in this paper to study the degree equitability of a graph by defining and studying some basic properties of degree equitable connectivity, degree equitable regularity, and degree equitable completeness of a graph. Some new families of graphs and some interesting results are obtained. In this paper for brevity we use equitable instead of degree equitable.

\section{Elementary Results}

Let $G=(V, E)$ be a graph. An equitable walk is defined as a finite alternating sequence of vertices and equitable edges, beginning and ending with vertices, such that each equitable edge is incident with the vertices preceding and following it. No equitable edge appears (is covered or traversed) more than once in the equitable walk. A vertex, however, may appear more than once. An equitable walk which begin and end at the same vertex called closed equitable walk. An equitable walk is not closed if the terminal vertices are distinct. An open equitable walk in which no vertex appears more than once is called an equitable path. The number of edges in an equitable path is called the length of the equitable path. A closed equitable walk in which no vertex (except the initial and the final vertex) appears more than once is called an equitable circuit.

Now, we prove some results representing the relations between the sum of the equitable degree of the vertices, the number of edges and the number of equitable edges.

Theorem 2.1. For any graph $G=(V, E)$ with $p$ vertices $v_{1}, v_{2}, \cdots, v_{p}$ and $q$ edges, $\sum_{i=1}^{p} \operatorname{deg}_{e}\left(v_{i}\right) \leq 2 q$.

Further, the equality hold if and only if every edge in $G$ is equitable edge.

Proof. We have $N_{e}(v) \subseteq N(v)$, for any vertex $v$ in a graph $G$. Then it is clear that $\left|N_{e}(v)\right| \leq|N(v)|$ and we have:

$$
\sum_{i=1}^{p} \operatorname{deg}\left(v_{i}\right)=\sum_{i=1}^{p}\left|N\left(v_{i}\right)\right|=2 q
$$

similarly,

$$
\sum_{i=1}^{p} \operatorname{deg}_{e}\left(v_{i}\right)=\sum_{i=1}^{p}\left|N_{e}\left(v_{i}\right)\right|,
$$

which implies

$$
\sum_{i=1}^{p} \operatorname{deg}_{e}\left(v_{i}\right)=\sum_{i=1}^{p}\left|N_{e}\left(v_{i}\right)\right| \leq \sum_{i=1}^{p}\left|N\left(v_{i}\right)\right| .
$$

Hence $\sum_{i=1}^{p} \operatorname{deg}_{e}\left(v_{i}\right) \leq 2 q$. Further, if every edge in $G$ is equitable edge, then $N_{e}(v)=N(v)$ for any vertex $v$ in $G$ that means $\sum_{i=1}^{p} \operatorname{deg}_{e}\left(v_{i}\right)=2 q$. The converse is obvious, if $\sum_{i=1}^{p} \operatorname{deg}_{e}\left(v_{i}\right)=2 q$, then every edge in $G$ is equitable edge.

For any graph $G$, the number of equitable edge denoted by $m_{e}$ is called the equitable size. The vertex $v \in V(G)$ is called equitable odd vertex (equitable even vertex) if $\operatorname{deg}_{e}(v)$ is odd number (even number).

Theorem 2.2. The sum of the equitable degrees of a graph is twice the number of equitable edges in it, that is $\sum_{i=1}^{p} \operatorname{deg}_{e}\left(v_{i}\right)=2 m_{e}$.

Proof. Let $G$ be a graph. Then any equitable edge contributes to the equitable degrees of two distinct vertices. Thus when the equitable degrees of the vertices are added, each equitable edge is counted exactly two times. Thus the sum of the equitable degrees is twice the equitable size of the graph, that is $\sum_{i=1}^{p} \operatorname{deg}_{e}\left(v_{i}\right)=2 m_{e}$.

Theorem 2.3. Every graph has an even number of equitable odd vertices.

Proof. Suppose that the sum of the equitable degrees of the equitable odd vertices is $x$ and the sum of the equitable degrees of the equitable even vertices is $y$. The number $y$ is even, and the number $x+y=2 m_{e}$ is even. Hence $x$ is even. If there are $t$ equitable odd vertices, the even number $x$ is the sum of $t$ odd numbers. So $t$ is even.

We can define the equitable complete graph $G$ as a connected graph which all its edges are equitable edges. and analogous to the equitable complete graph we can defined the equitable complement graph of a graph $G$ as following:

Definition 2.4. For any graph $G$, the equitable complement graph of $G$ denoted by $\bar{G}^{e}$ is the graph with the same vertices as $G$ and any two vertices $u, v$ are adjacent if $u$ and $v$ are not equitable adjacent in $G$.

The relation between the complement of the graph and the equitable complement graph of a graph can be found in the following theorem.

Theorem 2.5. For any graph $G, \bar{G} \subseteq \bar{G}^{e}$.

Proof. Let $f=u v$ be any edge in $\bar{G}$. Then $u$ and $v$ are not-adjacent vertices in $G$, which implies $u$ and $v$ are not equitable adjacent in $G$. Hence $e=u v$ is an edge in the graph $\bar{G}^{e}$. Therefore $\bar{G} \subseteq \bar{G}^{e}$.

Theorem 2.6. For any graph $G$ with $p$ vertices, $\bar{G}^{e} \cong \bar{G}$ if and only if $G$ is isomorphic to an equitable complete Graph.

Proof. Let $G$ be an equitable complete Graph and let $f=u v$ be any edge in the graph $\bar{G}^{e}$. Then $u$ is nonadjacent to $v$ in $G$. Hence $e=u v$ is an edge in $\bar{G}$, and since from the previous theorem we have $\bar{G} \subseteq \bar{G}^{e}$. 
Hence $\bar{G}^{e} \cong \bar{G}$. Conversely, suppose that $\bar{G}^{e} \cong \bar{G}$ and $G$ is not an equitable complete graph. Then there exists at least one edge $e=u v$ in $G$ which is not equitable edge. That means $u$ and $v$ are not equitable adjacent, which implies that $e=u v$ is an edge in $\bar{G}^{e}$. But clearly $u$ and $v$ are not adjacent in $\bar{G}$, a contradiction.

A graph $G$ is called an equitable edge-free graph if for any two adjacent vertices $u$ and $v$ in $G$, $|\operatorname{deg}(u)-\operatorname{deg}(v)| \geq 2$.

Proposition 2.7. For any graph $G$ with $p$ vertices, $\bar{G}^{e} \cong K_{p}$ if and only if $G$ is an equitable edge-free.

Proof. Let $G$ be an equitable edge-free graph with $p$ vertices. Then any two vertices in $\bar{G}^{e}$ are adjacent. Hence $\bar{G}^{e}=K_{p}$.

Conversely, suppose $\bar{G}^{e}=K_{p}$. Hence any two vertices in $\bar{G}^{e}$ are adjacent, then $G$ has no any equitable edge. Therefore $G$ is equitable edge-free.

Theorem 2.8. For any graph $G$ with $p$ vertices, $\bar{G}^{e} \cong \overline{K_{p}}$ if and only if $G$ is isomorphic to the complete Graph $K_{p}$.

Proof. Let $G \cong K_{p}$. Then any two vertices $u, v \in V(G)$ are equitable adjacent. Hence $\bar{G}^{e}$ does not contain any edge. Therefore $\bar{G}^{e} \cong \overline{K_{p}}$.

Conversely, suppose that $\bar{G}^{e} \cong \overline{K_{p}}$, and if possible $G$ is not complete Graph. This implies that there exist at least two nonadjacent vertices $u$ and $v$ in $G$ which are adjacent in $\bar{G}^{e}$. Thus $u$ and $v$ are adjacent in $\overline{K_{p}}$, a contradiction. Hence $G$ is complete graph $K_{p}$.

Corollary 2.9. For any equitable edge-free graph $G$ with $p$ vertices, $\overline{\bar{G}}^{e} \cong G$.

Theorem 2.10. Let $G=(V, E)$ be a graph with $p$ vertices and contains at least one non equitable edge with the property that any two vertices $u, v$ in $G$, we have $N_{e}[u] \cup N_{e}[v]=V(G)$. Then $\overline{\bar{G}}^{e} \cong K_{p}$.

Proof. Let $u, v$ be any two vertices in $G$. Then we have two cases:

Case 1: If $u$ and $v$ are adjacent vertices in $G$, then we have two subcases:

a) If $u v$ is equitable edge, then $u$ and $v$ are ${ }_{e}$ not adjacent in $\bar{G}^{e}$. Thus $u$ and $v$ are adjacent in $\overline{\bar{G}}^{e}$.

b) If $u v$ is not equitable edge that means $N_{e}(u) \cap N_{e}(v)=\varphi$ and since $N_{e}[u] \cup N_{e}[v]=V(G)$. Then $u$ and $v$ are adjacent but not equitable adjacent in $\bar{G}^{e}$. Therefore, $u$ and $v$ are adjacent in $\overline{\bar{G}}^{e}$.

Case 2: If $u$ and $v$ are nonadjacent vertices in $G$, then $u$ and $v$ are adjacent vertices in $\bar{G}^{e}$. Since $N_{e}[u] \cup N_{e}[v]=V(G)$, we get $u$ and $v$ are not equitable adjacent in $\bar{G}^{e}$. Hence $u$ is adjacent to $v$ in $\overline{\bar{G}}^{e}$. Therefore any two vertices in $\overline{\bar{G}}^{e}$ are adjacent.
Hence $\overline{\bar{G}}^{e} \cong K_{p}$.

\section{Equitable Connectivity and Equitable Regularity}

A graph $G$ is said to be equitable connected if there is at least one equitable path between every pair of vertices in $G$. Otherwise, $G$ is equitable disconnected. It is easy to see that an equitable disconnected graph consists of two or more equitable connected graphs. Each of these equitable connected subgraphs is called equitable component. Clearly any equitable connected graph is connected but the converse is not true equitable in general. For example, the graph $K_{1, m}$, where $m \geq 3$ is connected but not equitable connected.

Theorem 3.1. The isomorphism between the graphs preserve the number of equitable component.

Proof. Let $G=(V, E)$ and $H=(W, F)$ be isomorphic graphs. Let $f: V \rightarrow W$ be an isomorphism. If $\left[v_{1}, v_{2}, \cdots, v_{r}\right]$ is an equitable path in $G$ from $u=v_{1}$ to $w=v_{r}$, then $\left[f\left(v_{1}\right), f\left(v_{2}\right), \cdots, f\left(v_{r}\right)\right]$ is an equitable path in $H$ from $f(u)$ to $f(w)$. Thus, $u$ and $w$ are in the same equitable component of $G$ if and only if $f(u)$ to $f(w)$ are in the same component of $H$.

Theorem 3.2. A graph $G$ is equitable disconnected if and only if its vertex set $V$ can be partitioned into two nonempty, disjoint subsets $V_{1}$ and $V_{2}$ such that there exists no equitable edge in $G$ whose one end vertex is in subset $V_{1}$ and the other in subset $V_{2}$.

Proof. Suppose that we have the partition of $V(G)$ into disjoint subsets $V_{1}$ and $V_{2}$ such that there exists no equitable edge in $G$ whose one end vertex is in subset $V_{1}$ and the other in subset $V_{2}$. Consider two arbitrary vertices $a$ and $b$ of $V(G)$, such that $a \in V_{1}$ and $b \in V_{2}$. Then there is no equitable path between the vertices $a$ and $b$. Hence, if a partition exists, $G$ is not equitable connected.

Conversely, let $G$ be a disconnected graph. Consider $a$ to be a vertex in $G$. Let $V_{1}$ be the set of all vertices that are joined by equitable paths to $a$. Since $G$ is equitable disconnected, $V_{1}$ does not include all vertices of $G$. The remaining vertices will form a (nonempty) set $V_{2}$. No vertex in $V_{1}$ is joined to any in $V_{2}$ by an equitable edge. Hence the partition.

Theorem 3.3. If a graph (equitable connected or equitable disconnected) has exactly two vertices of odd equitable degree, then there exists an equitable path joining these two vertices.

Proof. Let $G$ be a graph with all even vertices except vertices $v_{1}$ and $v_{2}$, which are odd. From Theorem 2.3, no graph can have an odd number of equitable odd vertices. Therefore, in graph $G, v_{1}$ and $v_{2}$ must belong to the same equitable component, and hence must have equitable path between them. 
Definition 3.4. Let $G$ be a graph on $n>2$ vertices. An equitable disconnecting set of edges is a subset $D \subset E(G)$ such that $G-D$ is equitable disconnected. The edge equitable connectivity, $\varepsilon_{e}(G)$, is the smallest number of edges in any equitable disconnecting set.

We adopt the convention that $\varepsilon_{e}\left(K_{1}\right)=0$. Thus $\varepsilon_{e}(G)=0$ if and only if $G=K_{1}$ or $G$ is equitable disconnected. If $\varepsilon_{e}(G)=1$ then $G$ is a connected graph having an equitable edge $f$ such that $G-f$ is equitable disconnected. An equitable edge whose removal increases the number of equitable components is called equitable cut-edge (equitable bridge) of $G$.

Example. In the graph $G$ in Figure 1, the edge (12) is equitable cut edge but not cut edge. So we have $\varepsilon_{e}(G)=1$ but $\varepsilon(G)=2$.

Proposition 3.5. If $G$ is a graph, then $\varepsilon_{e}(G) \leq \delta_{e}(G)$. That is, the edge equitable connectivity of $G$ can be no larger than the minimum equitable degree of $G$.

Proof. let $G$ be an equitable connected graph on $n>2$ vertices and suppose $u \in V(G)$ is a vertex of equitable degree $\operatorname{deg}_{e}(u)=\delta_{e}(G)>0$. Since

$D=\left\{u v: v \in N_{e}(u)\right\}$ is an equitable disconnecting set of edges, $\delta_{e}(G)=|D| \geq \varepsilon_{e}(G)$.

The following result is straightforward.

Proposition 3.6. Suppose $G$ is an equitable connected graph. Let $e=u v$ be equitable edge. Then $e$ is an equitable cut-edge of $G$ if and only if $P=[u, v]$ is the only equitable path in $G$ from $u$ to $v$.

Definition 3.7. Let $G$ be an equitable connected graph. An equitable vertex cut (or an equitable separateing set) of $G$ is a set $S \subset V(G)$ such that $G-S$ is equitable disconnected. The connectivity, $k_{e}(G)$, is the smallest number of vertices in any equitable vertex cut of $G$. A vertex whose removal increases the number of equitable components of $G$ is called equitable cut-vertex (or point of equitable articulation). For the graph in Figure 1, $k_{e}(G)=1$ but $k(G)=2$.

The maximal equitable connected subgraph of $G$ that has no equitable cut-vertex is called an equitable block of $G$.

Theorem 3.8. For any graph $G, k_{e}(G) \leq \varepsilon_{e}(G)$.

Proof. If $\varepsilon_{e}(G)=0$, then $G$ is equitable disconnected and $k_{e}(G)=\varepsilon_{e}(G)=0$. If $\varepsilon_{e}(G)=1$, then this

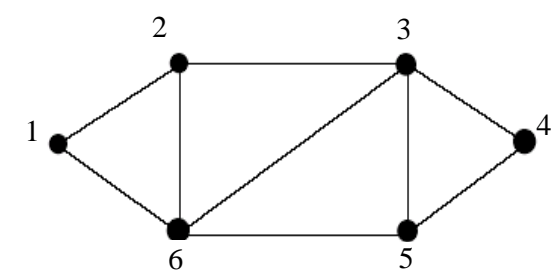

Figure 1. Equitable cut edge but not cut edge. graph is equitable connected with equitable bridge $x$, that means $G=K_{2}$ or one of the vertices which incident with $x$ is equitable cut vertex. Therefore, $k_{e}(G)=1$. If $\varepsilon_{e}(G) \geq 2$, then removal $\varepsilon_{e}(G)$ edges results in equitable disconnected graph, that means the removal $\varepsilon_{e}(G)-1$ of this edges results in a graph $G$ with an equitable bridge $x=u v$. For each of these $\varepsilon_{e}(G)-1$ edges select an incident vertex different from $u$ or $v$. The removal of these $\varepsilon_{e}(G)-1$ vertices remove all the $\varepsilon_{e}(G)-1$ edges. If the resulting graph is disconnected, then $k_{e}(G) \leq \varepsilon_{e}(G)$. If not, $x$ is equitable bridge of this subgraph and hence the removal of $u$ or $v$ results in an equitable disconnected. Hence, $k_{e}(G) \leq \varepsilon_{e}(G)$.

Definition 3.9. A graph $G$ is called $k$-equitable regular graph if $\Delta_{e}(G)=\delta_{e}(G)=k$.

Observation 3.10. Every $k$-regular graph is $k$-equitable regular graph but the converse is not true, in general.

Example. The graph in the Figure 2 is 2-equitable regular graph but not regular.

\section{An Equitable Line Graph and an Equitable Total Graph}

Definition 4.1. Given a graph $G$, its equitable line graph $L_{e}(G)$ is a graph such that

1) Each vertex of $L_{e}(G)$ represents an equitable edge of $G$; and

2) Two vertices of $L_{e}(G)$ are adjacent if and only if their corresponding equitable edges share a common endpoint (are adjacent) in $G$.

Proposition 4.2. The line graph of equitable connected graph is connected.

Proof. If $G$ is equitable connected, it contains equitable path connecting any two of its edges, which translates into a path in $L_{e}(G)$ containing any two of the vertices of $L_{e}(G)$. Hence $L_{e}(G)$ is connected.

Observation 4.3. Let $G$ be any graph. Then $L_{e}(G) \subseteq L(G)$.

Remark. The equitable line graph of equitable connected graph is connected but not equitable connected in general. In Figure 3, the equitable line graph of equitable connected graph is connected but not equitable connected.

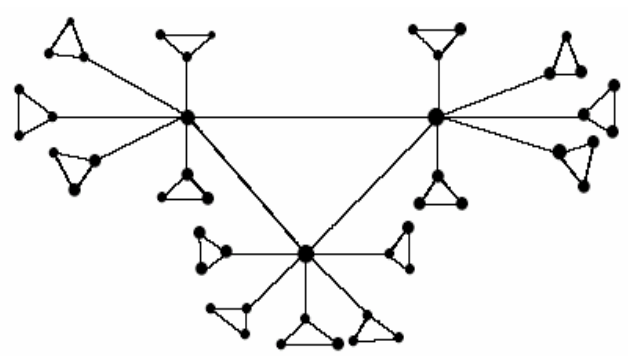

Figure 2. 2-equitable regular graph but not regular. 


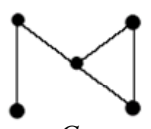

G

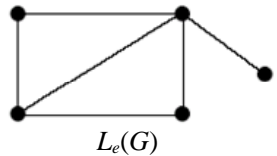

Figure 3. Equitable line graph of equitable connected graph.

Definition 4.4. Let $G=(V, E)$ be a graph. The equitable graph $G_{e}$ of $G$ is defined as the graph with vertex set $V(G)$ and two vertices $u, v$ are adjacent if and only if $u$ and $v$ are equitable adjacent in $G$.

The following result is immediate.

Proposition 4.5. For any graph $G$ with at least one equitable edge, the following hold.

1) $L_{e}(G)=L\left(G_{e}\right)$.

2) $L_{e}(G)=L(G)$ if and only if $G$ is an equitable complete graph.

3) If $G_{1} \cong G_{2}$, then $L_{e}\left(G_{1}\right) \cong L_{e}\left(G_{2}\right)$ but the converse is not true.

Theorem 4.6. If $G=(V, E)$ is a graph and $|V|=p$, $|E|=q$ whose vertices have equitable degree $\left(d_{e}\right)_{i}$, then $L_{e}(G)$ has $q_{e}$ vertices and $q_{L_{e}}=-q_{e}+\frac{1}{2} \sum\left(\left(d_{e}\right)_{i}\right)^{2}$, where $q_{e}$ is the number of equitable edges in $G$.

Proof. Clearly from the definition of equitable line graph $L_{e}(G)$ has $q_{e}$ vertices the $\left(d_{e}\right)_{i}$ equitable edges incident with a point $v_{i}$ contribute $\left(\begin{array}{c}\left(d_{e}\right)_{i} \\ 2\end{array}\right)$ to $q_{e}$, so

$$
\begin{aligned}
q_{L_{e}} & =\sum\left(\begin{array}{c}
\left(d_{e}\right)_{i} \\
2
\end{array}\right)=\frac{1}{2} \sum\left(d_{e}\right)_{i}\left(\left(d_{e}\right)_{i}-1\right) \\
& =-q_{e}+\frac{1}{2} \sum\left(\left(d_{e}\right)_{i}\right)^{2}
\end{aligned}
$$

Proposition 4.7. If $G=(V, E)$ is a graph, then $L_{e}(G) \cong L(G)$ if and only if $G$ is an equitable complete graph.

Proof. If $G$ is an equitable complete graph then clearly $L_{e}(G) \cong L(G)$.

Conversely, let $L_{e}(G) \cong L(G)$. Then $L_{e}(G)$ and $L(G)$ have the same number of vertices that means $q_{e}=q$. Hence $G$ is an equitable complete graph.

Theorem 4.8. A connected graph is isomorphic to its equitable line graph if and only if it is a cycle.
Proof. If $G$ is cycle, then clearly $G \cong L_{e}(G)$. Conversely, if $G$ is isomorphic to $L_{e}(G)$, that means $G$ is an equitable complete graph, and by Proposition 4.7. $L_{e}(G) \cong L(G)$. Hence $G \cong L(G)$. Therefore $G$ is cycle.

Definition 4.9. The equitable total graph $T_{e}=T_{e}(G)$ of a graph $G$ is a graph such that

1) The vertex set of $T_{e}$ corresponds to the vertices and equitable edges of $G$ and

2) Two vertices are adjacent in $T_{e}$ if and only if their corresponding elements are either adjacent or incident in $G$.

Observation 4.10. For any graph $G$ the equitable total graph is a subgraph of the total graph of a graph $G$.

Proposition 4.11. If $v$ is a vertex and $x=u w$ be equitable edge in a graph $G$, then the degree of the vertex $v$ is $d(v)+d_{e}(v)$ and the degree of $x$ is $d_{e}(u)+d_{e}(w)$ in $T_{e}(G)$.

Proposition 4.12. If $G=(V, E)$ is a graph with $p$ vertices and $q$ edges and its vertices have equitable degree $\left(d_{e}\right)_{i}$, then $T_{e}(G)$ has $p+q_{e}$ vertices and

$q_{T_{e}}=q_{e}+q+\frac{1}{2} \sum\left(\left(d_{e}\right)_{i}\right)^{2}$ where $q_{e}$ is the number of equitable edges in $G$.

Proof. The number of vertices of the graph $T_{e}(G)$ is the sum of number of equitable edge and number of vertices in $G$, that is $T_{e}(G)$ has $p+q_{e}$ vertices. By the definition of total graph of $G$, the number of edges in $T_{e}(G)$ is the sum of edges in $G$ and the number of edges in the equitable line graph of $G$ and twice the number equitable edges in $G$, that is;

$$
\begin{aligned}
q_{T_{e}} & =2 q_{e}+q-q_{e}+\frac{1}{2} \sum\left(\left(d_{e}\right)_{i}\right)^{2} . \text { Hence, } \\
q_{T_{e}} & =q_{e}+q+\frac{1}{2} \sum\left(\left(d_{e}\right)_{i}\right)^{2} .
\end{aligned}
$$

\section{REFERENCES}

[1] F. Harary, “Graph Theory,” Addison-Wesley, Reading, 1969.

[2] V. Swaminathan and K. M. Dharmalingam, "Degree Equitable Domination on Graphs," Kragujevac Journal of Mathematics, Vol. 35, No. 1, 2011, pp. 191-197. 